\title{
Prevalence of overweight and obesity among migrants in Switzerland: association with country of origin
}

\author{
Pedro Marques-Vidal ${ }^{1,2, *}$, Peter Vollenweider ${ }^{3}$, Gérard Waeber ${ }^{3}$ and Fred Paccaud ${ }^{1}$ \\ ${ }^{1}$ Institute of Social and Preventive Medicine (IUMSP), University Hospital (CHUV), Faculty of Biology and \\ Medicine of Lausanne, Bugnon 17, 1005 Lausanne, Switzerland: ${ }^{2}$ Clinical Research Centre, University Hospital \\ (CHUV), Lausanne, Switzerland: ${ }^{3}$ Department of Medicine, University Hospital (CHUV), Lausanne, Switzerland
}

Submitted 12 October 2010: Accepted 9 January 2011: First published online 22 February 2011

\begin{abstract}
Objective: Migrants tend to present higher overweight and obesity levels, but whether this relationship applies to all nationalities has seldom been studied. The present study aimed to assess the prevalence of overweight and obesity according to nationality in adults.

Design: Cross-sectional population-based samples.

Setting: Five-year nationwide interview surveys (Swiss Health Surveys - SHS) from 1992 to 2007 ( $n$ 63 766) and a local examination survey (CoLaus Study in Lausanne 2004-2006, $n$ 6743).

Subjects: Participants were separated into Swiss, French, German, Italian, Portuguese, Spanish nationals, those from the former Republic of Yugoslavia and from other European and other countries.

Results: Compared with Swiss nationals, German and French nationals presented a lower prevalence of overweight and obesity, whereas nationals from Italy, Spain, Portugal and the former Republic of Yugoslavia presented higher levels. Adjusting the SHS data for age, gender, education, smoking, leisure-time physical activity and survey year, a lower risk for overweight and obesity was found for German (OR = 0.80, 95\% CI 0.70, 0.92) and French (OR=0.74, 95\% CI 0.61, 0.89) nationals, whereas higher risks were found for participants from Italy (OR $=1 \cdot 45,95 \%$ CI 1.33, $1 \cdot 58)$, Spain (OR $=1 \cdot 36,95 \%$ CI 1·15, 1·61), Portugal (OR $=1 \cdot 25,95 \%$ CI 1·06, 1·47) and the former Republic of Yugoslavia $(\mathrm{OR}=1 \cdot 98,95 \% \mathrm{CI} 1 \cdot 69,2 \cdot 32)$. Similar findings were observed in the CoLaus Study for Italian (OR $=1 \cdot 63$, 95\% CI $1 \cdot 29$, $2 \cdot 06)$, Spanish (OR $=1 \cdot 54,95 \%$ CI 1·17, 2·04) and Portuguese (OR $=1 \cdot 49$, 95\% CI $1 \cdot 16,1 \cdot 91)$ participants and for those from the former Republic of Yugoslavia (OR $=5 \cdot 34,95 \%$ CI 3·00, 9·50).

Conclusions: Overweight and obesity are unevenly distributed among migrants in Switzerland. Migrants from Southern Europe and from the former Republic of Yugoslavia present higher prevalence rates. This suggests that preventive messages should be tailored to these specific populations.
\end{abstract}

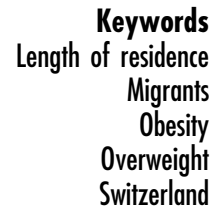

Obesity has reached pandemic proportions. In Europe it is estimated that $30-80 \%$ of adults are overweight or obese, with approximately 150 million individuals being obese $^{(1)}$. Several studies have shown that migrants present a higher prevalence of overweight and obesity than nationals ${ }^{(2-4)}$, although this finding has not been found in other studies ${ }^{(5,6)}$. Switzerland has a large migrant community of over 1.6 million, representing over one-fifth of the total population ${ }^{(7)}$. Nevertheless, the prevalence of overweight and obesity according to migrant status or nationality of adult migrants in Switzerland has seldom been studied ${ }^{(8)}$.

Hence, we used the data from two large, populationbased samples (Swiss Health Surveys (SHS) and CoLaus
Study) to assess the prevalence of overweight and obesity according to nationality among adults in Switzerland.

\section{Experimental methods}

\section{CoLaus Study}

The CoLaus Study was approved by the Institutional Ethics Committee of the University of Lausanne. The CoLaus Study is cross-sectional in nature aimed at assessing the prevalence and deciphering the molecular determinants of cardiovascular risk factors in the Caucasian population of Lausanne, Switzerland, a town of 117161 inhabitants, of whom 79420 are of Swiss nationality. 
The sampling procedure of the CoLaus Study has been described previously ${ }^{(9)}$. Briefly, the complete list of Lausanne inhabitants aged 35-75 years ( $n$ 56694) was provided by the population registry of the city. A simple, non-stratified random sample of $35 \%$ of the overall population was drawn. The following inclusion criteria were applied: (i) written informed consent; (ii) 35-75 years of age; (iii) willingness to participate in the study and donate a blood sample; and (iv) Caucasian origin. Recruitment began in June 2003 and ended in May 2006. Participation rate was $41 \%$. For the present study, data from non-Caucasian participants ( $n$ 555), initially excluded from the main study but assessed the same way, were also included.

All participants presented to the outpatient clinic of the University Hospital of Lausanne in the morning after an overnight fast. Data were collected by trained field interviewers in a single visit lasting about $60 \mathrm{~min}$.

\section{Swiss Health Survey}

Data from the four SHS were obtained from the Swiss Federal Statistical Office (www.bfs.admin.ch). The SHS is a cross-sectional, nationwide, population-based telephonic survey conducted every 5 years since 1992 by the Swiss Federal Statistical Office under a mandate of the Federal Government ${ }^{(10)}$. The SHS aims to track public health trends in a representative sample of the resident population of Switzerland aged $\geq 15$ years ${ }^{(11)}$. To date, the survey has been carried out four times, in 1992-1993, 1997, 2002 and 2007.

The study population was chosen by stratified random sampling of a database of all private Swiss households with fixed line telephones. The first sampling stratum consisted of the seven main regions: West 'Leman', West-Central 'Mittelland', Northwest, Zurich, North-East, Centre and South. The second stratum consisted of the cantons, and the number of households drawn was proportional to the population of the canton. In some cantons, households were oversampled to obtain accurate cantonal estimates. The third stratum consisted of households. One member of the household was randomly selected in advance from all members aged $\geq 15$ years. A letter inviting this selected household member to participate in the survey was sent to each sampled individual, who was then contacted thereafter by phone and interviewed using a computer-assisted telephone interview software to manage dialling and data collection. Face-to-face interviews were organized for participants older than 75 years. In the case of long-term absence of a sampled individual, a proxy interviewee was requested to provide answers on behalf of the predefined sampled person (it is estimated that this substitution occurred in approximately $3 \%$ of households). The interviews were carried out in German, French or Italian, as appropriate. People who did not speak any of these three languages were excluded from the survey. Other criteria for exclusion were: asylum seeker status; households without a fixed line telephone; very poor health status; and living in a nursing home $^{(12)}$. Participation rate was $71 \%$ in $1992-1993,85 \%$ in 1997, 64\% in 2002 and $66 \%$ in 2007. It is estimated that $<2 \%$ of households were excluded because of these exclusion criteria. More details are available at http://www. bfs.admin.ch/bfs/portal/fr/index/infothek/erhebungen_ quellen/blank/blank/ess/01.html.

\section{Data collected}

The following nationalities were considered: Swiss, former Yugoslavian, French, German, Italian, Portuguese and Spanish. Owing to the small number of participants, the other nationalities were grouped as follows: other European nations and the rest of the world. The choice was based on a previous study ${ }^{(8)}$, although we did not consider Turkish migrants because of small sample sizes. When a participant had dual nationality, the Swiss or the first given nationality (if not Swiss) was considered. For the CoLaus Study, the country of birth was considered as the nationality, whereas for the SHS the participants were directly asked about their nationality. The length of residence in Switzerland was assessed for all participants in the CoLaus Study, whereas only the SHS for 2007 collected such information.

In the CoLaus Study, body weight and height were measured with participants standing without shoes in light indoor clothes. Body weight was measured in kilograms to the nearest $100 \mathrm{~g}$ using a Seca ${ }^{\circledR}$ scale (Seca Schweiz, Reinach, Switzerland), which was calibrated regularly. Height was measured to the nearest $5 \mathrm{~mm}$ using a Seca ${ }^{\circledR}$ height gauge (Seca Schweiz). In the SHS, the participants were asked about their current body weight and height. BMI was calculated as weight in kilograms divided by the square of height in metres $\left(\mathrm{kg} / \mathrm{m}^{2}\right)$. Participants were considered to be normal weight, overweight or obese if their BMI was $<25 \cdot 0, \geq 25 \cdot 0$ and $<30 \cdot 0$ or $\geq 30 \cdot 0 \mathrm{~kg} / \mathrm{m}^{2}$, respectively.

Waist was measured with a non-stretchable tape over the unclothed abdomen at the narrowest point between the lowest rib and the iliac crest ${ }^{(13)}$. Two measurements were recorded and the mean (expressed in centimetres) was used for analyses. Abdominal obesity was defined as waist circumference $\geq 102 \mathrm{~cm}$ for men and $\geq 88 \mathrm{~cm}$ for women ${ }^{(13)}$.

Three age categories were considered: 18-34, 35-64 and $\geq 65$ years. Education was categorized as follows: (i) no education completed/primary school (referred to as 'basic'); (ii) apprenticeship/secondary level (referred to as 'secondary'); and (iii) tertiary level, which included university and other forms of education after the secondary level (referred to as 'university'). Leisure-time physical activity was considered when the participant reported exercising at least once per week; no answer was considered as a negative answer. Smoking status was divided into current, former (irrespective of the delay) and never. 


\section{Statistical analysis}

Statistical analysis was conducted using the SAS statistical software package version 9.2 (SAS Institute, Cary, NC, USA). For the SHS, a first analysis was conducted using the original data. A second analysis was conducted after weighting each participant. Weights were computed taking into account the percentage of non-responders by raking ratio estimation ${ }^{(14)}$. Weighting partly allows the correction for bias, i.e. participants with given characteristics who are under-represented in the original sample are attributed a higher weight.

Quantitative variables were expressed as mean and SD and qualitative variables as number of participants and percentage. Continuous and categorical data were analysed using the $t$ test and the $\chi^{2}$ test, respectively. The relationships between length of residence and BMI were assessed by Pearson's correlation and multiple regression, adjusting for age; the results of the regression analysis were expressed as standardized coefficients. Standardized regression coefficients represent the change in the dependent variable, expressed as a fraction of the SD per SD change in the independent variable, and can be considered as adjusted correlation coefficients. In the SHS, the time trends (1992-2007) in BMI were also assessed separately for each nationality by multiple regression adjusting for age and sex, and the results were expressed as standardized coefficients. As the number of participants with obesity according to nationality and survey year was relatively small, the combined prevalence of overweight and obesity was used, and its time trend was assessed separately for each nationality using the Cochran-Armitage test.

The impact of nationality on the risk of presenting with overweight and/or obesity was assessed by multivariate logistic regression analysis adjusting for age, gender, educational level, smoking status and physical activity. For the SHS, a further adjustment on survey year was applied. The results were expressed as OR and 95\% CI. Statistical significance was considered for $P<0 \cdot 05$.

\section{Results}

\section{Sample characteristics}

Overall, 63766 participants from the SHS and 6743 participants from the CoLaus Study (6188 from the main study and 555 non-Caucasians) were included in the analyses. Their demographic and anthropometric characteristics according to nationality are summarized in Table 1. In both samples, participants from Southern Europe and from the former Republic of Yugoslavia were younger, had a lower educational level, practised leisure-time physical activity less frequently, smoked more and were living in Switzerland for a shorter time. The proportions of women were also lower among German, Italian and Spanish citizens.

\section{Overweight and obesity levels}

Differing prevalence of overweight and obesity was found according to nationality (Table 1). Compared with Swiss nationals, German and French nationals presented lower levels of overweight and obesity, whereas Southern European nationals and citizens from the former Republic of Yugoslavia presented higher levels. Similar findings were obtained for waist and abdominal obesity (Table 1). On multivariate analysis adjusting for age, gender, education, smoking, leisure-time physical activity and survey year (for the SHS only), German and French nationals tended to have a lower risk, whereas participants from Southern European countries (Italy, Spain, Portugal) and from the former Republic of Yugoslavia had a higher risk of presenting with overweight or obesity (Table 2).

The length of residence of non-Swiss nationals was positively related with BMI (Pearson $r=0 \cdot 203, n 1853$ and $r=0 \cdot 089, n 2640$, for the SHS and the CoLaus Study, respectively, both with $P<0 \cdot 001)$. Findings were similar for waist circumference (Pearson $r=0 \cdot 126, P<0 \cdot 001$ ) in the CoLaus Study. These significant positive relationships remained after adjusting for age in SHS 2007 (standardized regression coefficient $=0 \cdot 11, P<0 \cdot 001$ ), but not in the CoLaus Study (standardized regression coefficient $=$ $-0.03, \quad P=0.31$ and $-0.02, P=0.38$, respectively). Splitting the length of residence into 10 -year categories $(<10,10-19,20-29$ and $\geq 30$ years) showed no significant relationship with obesity and overweight/obesity after adjusting for age, gender, smoking, leisure-time physical activity and nationality in the CoLaus Study, and no clear trend was identified in the SHS 2007 (Table 3). As for BMI-derived obesity, no relationship between length of residence and abdominal obesity was found, and no differences were found regarding nationality; for French and Portuguese nationals, OR $=0.74$ (95\% CI $0 \cdot 44,1 \cdot 25)$ and $0 \cdot 80(95 \%$ CI $0 \cdot 45,1 \cdot 43)$, respectively $(P=\mathrm{NS})$.

After adjusting for age and sex, positive increases in BMI with time were found for German (standardized regression coefficient $=0 \cdot 065, P<0 \cdot 02$ ), Italian (standardized regression coefficient $=0.057, \quad P<0.004)$, French (standardized regression coefficient $=0 \cdot 095, \quad P<0 \cdot 01$ ), Portuguese (standardized regression coefficient $=0 \cdot 113$, $P<0 \cdot 002$ ), other European (standardized regression coefficient $=0.090, P<0 \cdot 001$ ) and other world (standardized regression coefficient $=0 \cdot 100, P<0 \cdot 004$ ) nationals, whereas no significant trends were found for Spanish (standardized regression coefficient $=0 \cdot 058, P=0 \cdot 14$ ) nationals and for those from the former Republic of Yugoslavia (standardized regression coefficient $=0 \cdot 065, P=0 \cdot 06)$. The corresponding value for Swiss nationals was $0 \cdot 048(P<0 \cdot 001)$. Similarly, the combined prevalence of overweight and obesity increased with time in most migrant groups, with the exception of Spain and the former Republic of Yugoslavia (Table 4). 
Table 1 Characteristics of the participants according to nationality: SHS 1992-2007 and the CoLaus Study

\begin{tabular}{|c|c|c|c|c|c|c|c|c|c|c|c|c|c|c|c|c|c|c|}
\hline \multirow[b]{2}{*}{ Characteristic } & \multicolumn{2}{|c|}{ Swiss } & \multicolumn{2}{|c|}{ French } & \multicolumn{2}{|c|}{ German } & \multicolumn{2}{|c|}{ Italian } & \multicolumn{2}{|c|}{ Portuguese } & \multicolumn{2}{|c|}{ Spanish } & \multicolumn{2}{|c|}{$\begin{array}{l}\text { Former Republic } \\
\text { of Yugoslavia }\end{array}$} & \multicolumn{2}{|c|}{ Other European } & \multicolumn{2}{|c|}{ Other } \\
\hline & $\begin{array}{l}n \text { or } \\
\text { Mean }\end{array}$ & $\begin{array}{c}\% \text { or } \\
\text { SD }\end{array}$ & $\begin{array}{l}n \text { or } \\
\text { Mean }\end{array}$ & $\begin{array}{l}\% \text { or } \\
\text { SD }\end{array}$ & $\begin{array}{l}n \text { or } \\
\text { Mean }\end{array}$ & $\begin{array}{l}\% \text { or } \\
\text { SD }\end{array}$ & $\begin{array}{l}n \text { or } \\
\text { Mean }\end{array}$ & $\begin{array}{l}\% \text { or } \\
\text { SD }\end{array}$ & $\begin{array}{l}n \text { or } \\
\text { Mean }\end{array}$ & $\begin{array}{c}\% \text { or } \\
\text { SD }\end{array}$ & $\begin{array}{l}n \text { or } \\
\text { Mean }\end{array}$ & $\begin{array}{c}\% \text { or } \\
\text { SD }\end{array}$ & $\begin{array}{l}n \text { or } \\
\text { Mean }\end{array}$ & $\begin{array}{l}\% \text { or } \\
\text { SD }\end{array}$ & $\begin{array}{l}n \text { or } \\
\text { Mean }\end{array}$ & $\begin{array}{l}\% \text { or } \\
\text { SD }\end{array}$ & $\begin{array}{l}n \text { or } \\
\text { Mean }\end{array}$ & $\begin{array}{c}\% \text { or } \\
\text { SD }\end{array}$ \\
\hline \multicolumn{19}{|l|}{ SHS } \\
\hline Sample size & \multicolumn{2}{|c|}{ (n 55238) } & \multicolumn{2}{|c|}{$(n 646)$} & \multicolumn{2}{|c|}{$(n$ 1131) } & \multicolumn{2}{|c|}{$(n 2533)$} & \multicolumn{2}{|c|}{$(n 691)$} & \multicolumn{2}{|c|}{$(n 616)$} & \multicolumn{2}{|c|}{$(n 687)$} & \multicolumn{2}{|c|}{ (n 1433) } & $(n 7$ & 91) \\
\hline Age (years) & $49 \cdot 2$ & $17 \cdot 7$ & $44 \cdot 7$ & $15 \cdot 3$ & $47 \cdot 1$ & $15 \cdot 5$ & $45 \cdot 7$ & $16 \cdot 1$ & $35 \cdot 5$ & $9 \cdot 4$ & $40 \cdot 2$ & $13 \cdot 4$ & $37 \cdot 5$ & $10 \cdot 8$ & $43 \cdot 1$ & $15 \cdot 6$ & $37 \cdot 4$ & $11 \cdot 7$ \\
\hline Length of residence (years) ${ }^{\star}$ & - & - & $16 \cdot 6$ & $14 \cdot 7$ & $14 \cdot 9$ & $15 \cdot 5$ & $35 \cdot 3$ & $15 \cdot 2$ & $17 \cdot 0$ & $7 \cdot 6$ & $27 \cdot 7$ & $11 \cdot 8$ & $18 \cdot 0$ & $8 \cdot 1$ & $19 \cdot 3$ & $14 \cdot 9$ & $11 \cdot 0$ & $9 \cdot 0$ \\
\hline Women (\%) & 30812 & $55 \cdot 8$ & 346 & $53 \cdot 6$ & 576 & $50 \cdot 9$ & 1150 & $45 \cdot 4$ & 365 & $52 \cdot 8$ & 292 & $47 \cdot 4$ & 340 & $49 \cdot 5$ & 753 & $52 \cdot 6$ & 448 & $56 \cdot 6$ \\
\hline Educational level (\%) & & & & & & & & & & & & & & & & & & \\
\hline Basic & 9162 & $16 \cdot 6$ & 90 & $13 \cdot 9$ & 68 & $6 \cdot 0$ & 1085 & $42 \cdot 8$ & 475 & $68 \cdot 8$ & 277 & $45 \cdot 0$ & 233 & $34 \cdot 0$ & 325 & $22 \cdot 7$ & 214 & $27 \cdot 1$ \\
\hline Secondary & 34528 & $62 \cdot 5$ & 321 & $49 \cdot 7$ & 566 & $50 \cdot 0$ & 1216 & $48 \cdot 0$ & 200 & $28 \cdot 9$ & 283 & $45 \cdot 9$ & 385 & $56 \cdot 0$ & 637 & $44 \cdot 5$ & 338 & $42 \cdot 7$ \\
\hline University & 11548 & $20 \cdot 9$ & 235 & $36 \cdot 4$ & 497 & $44 \cdot 0$ & 232 & $9 \cdot 2$ & 16 & $2 \cdot 3$ & 56 & $9 \cdot 1$ & 69 & $10 \cdot 0$ & 471 & $32 \cdot 8$ & 239 & $30 \cdot 2$ \\
\hline Smoking status (\%) & & & & & & & & & & & & & & & & & & \\
\hline Never & 26702 & $48 \cdot 4$ & 231 & $35 \cdot 8$ & 509 & $45 \cdot 0$ & 1103 & $43 \cdot 6$ & 313 & $45 \cdot 3$ & 244 & $39 \cdot 6$ & 258 & $37 \cdot 6$ & 648 & $45 \cdot 2$ & 448 & $56 \cdot 6$ \\
\hline Former & 12004 & $21 \cdot 7$ & 169 & $26 \cdot 2$ & 266 & $23 \cdot 5$ & 538 & $21 \cdot 3$ & 137 & $19 \cdot 8$ & 121 & $19 \cdot 6$ & 104 & $15 \cdot 1$ & 300 & $20 \cdot 9$ & 116 & $14 \cdot 7$ \\
\hline Current & 16511 & $29 \cdot 9$ & 246 & $38 \cdot 1$ & 356 & 31.5 & 891 & $35 \cdot 2$ & 241 & $34 \cdot 9$ & 251 & $40 \cdot 8$ & 325 & $47 \cdot 3$ & 485 & 33.9 & 227 & $28 \cdot 7$ \\
\hline Leisure-time PA (\%) & 27264 & $49 \cdot 4$ & 273 & $42 \cdot 3$ & 657 & $58 \cdot 1$ & 824 & $32 \cdot 5$ & 178 & $25 \cdot 8$ & 189 & $30 \cdot 7$ & 214 & $31 \cdot 2$ & 592 & $41 \cdot 3$ & 276 & 34.9 \\
\hline BMI $\left(\mathrm{kg} / \mathrm{m}^{2}\right)$ & $24 \cdot 1$ & $4 \cdot 0$ & $23 \cdot 4$ & $3 \cdot 7$ & $23 \cdot 7$ & $3 \cdot 6$ & $25 \cdot 1$ & $4 \cdot 1$ & $24 \cdot 3$ & $3 \cdot 6$ & $24 \cdot 6$ & $3 \cdot 7$ & $25 \cdot 0$ & $3 \cdot 9$ & $24 \cdot 1$ & $4 \cdot 0$ & $23 \cdot 4$ & $4 \cdot 2$ \\
\hline BMI class (\%) & & & & & & & & & & & & & & & & & & \\
\hline Normal & 35381 & $64 \cdot 0$ & 471 & $72 \cdot 9$ & 799 & $70 \cdot 6$ & 1355 & $53 \cdot 5$ & 423 & $61 \cdot 2$ & 362 & $58 \cdot 8$ & 364 & $53 \cdot 0$ & 904 & $63 \cdot 1$ & 566 & $71 \cdot 6$ \\
\hline Overweight & 15670 & $28 \cdot 4$ & 140 & $21 \cdot 7$ & 277 & $24 \cdot 5$ & 907 & $35 \cdot 8$ & 228 & $33 \cdot 0$ & 214 & $34 \cdot 7$ & 247 & $36 \cdot 0$ & 421 & $29 \cdot 4$ & 183 & $23 \cdot 1$ \\
\hline Obese & 4187 & $7 \cdot 6$ & 35 & $5 \cdot 4$ & 55 & $4 \cdot 9$ & 271 & $10 \cdot 7$ & 40 & $5 \cdot 8$ & 40 & $6 \cdot 5$ & 76 & $11 \cdot 0$ & 108 & $7 \cdot 5$ & 42 & $5 \cdot 3$ \\
\hline The CoLaus Study & & & & & & & & & & & & & & & & & & \\
\hline Sample size & (n 404 ) & & (n 4 & 39) & $(n s$ & & (n 40 & & $(n 3$ & & (n) 2 & & & & & & (n 4 & 79) \\
\hline Age (years) & $54 \cdot 0$ & $10 \cdot 9$ & $51 \cdot 8$ & $10 \cdot 6$ & $55 \cdot 6$ & $11 \cdot 1$ & $56 \cdot 2$ & $10 \cdot 6$ & $44 \cdot 5$ & $6 \cdot 1$ & $51 \cdot 0$ & $8 \cdot 7$ & $45 \cdot 9$ & $7 \cdot 3$ & $50 \cdot 8$ & $10 \cdot 1$ & $48 \cdot 1$ & 8.9 \\
\hline Length of residence (years) & - & - & $27 \cdot 0$ & $15 \cdot 6$ & $33 \cdot 1$ & $12 \cdot 2$ & $36 \cdot 8$ & $11 \cdot 7$ & $19 \cdot 0$ & $7 \cdot 4$ & $29 \cdot 4$ & $10 \cdot 6$ & $19 \cdot 4$ & $9 \cdot 8$ & $23 \cdot 9$ & $13 \cdot 5$ & $21 \cdot 0$ & $12 \cdot 3$ \\
\hline Women (\%) & 2157 & $53 \cdot 3$ & 240 & $54 \cdot 7$ & 69 & $75 \cdot 0$ & 165 & $40 \cdot 8$ & 176 & $45 \cdot 0$ & 128 & $48 \cdot 9$ & 39 & $44 \cdot 8$ & 304 & $56 \cdot 0$ & 266 & $55 \cdot 5$ \\
\hline Educational level (\%) & & & & & & & & & & & & & & & & & & \\
\hline Basic & 526 & $13 \cdot 0$ & 53 & $12 \cdot 1$ & 9 & $9 \cdot 8$ & 183 & $45 \cdot 3$ & 303 & $77 \cdot 5$ & 135 & $51 \cdot 5$ & 34 & $39 \cdot 1$ & 89 & $16 \cdot 4$ & 86 & $18 \cdot 0$ \\
\hline Secondary & 2781 & $68 \cdot 7$ & 264 & $60 \cdot 1$ & 58 & $63 \cdot 0$ & 188 & $46 \cdot 5$ & 80 & $20 \cdot 5$ & 108 & $41 \cdot 2$ & 44 & $50 \cdot 6$ & 242 & $44 \cdot 6$ & 240 & $50 \cdot 1$ \\
\hline University & 739 & $18 \cdot 3$ & 122 & $27 \cdot 8$ & 25 & $27 \cdot 2$ & 33 & $8 \cdot 2$ & 8 & $2 \cdot 1$ & 19 & $7 \cdot \overline{3}$ & 9 & $10 \cdot 3$ & 212 & $39 \cdot 0$ & 153 & $31 \cdot 9$ \\
\hline Smoking status (\%) & & & & & & & & & & & & & & & & & & \\
\hline Never & 1589 & $39 \cdot 3$ & 166 & $37 \cdot 8$ & 35 & $38 \cdot 0$ & 149 & $36 \cdot 9$ & 177 & $45 \cdot 3$ & 118 & $45 \cdot 0$ & 36 & $41 \cdot 4$ & 280 & $51 \cdot 6$ & 196 & $40 \cdot 9$ \\
\hline Former & 1366 & $33 \cdot 8$ & 140 & $31 \cdot 9$ & 37 & $40 \cdot 2$ & 145 & 35.9 & 104 & $26 \cdot 6$ & 81 & $30 \cdot 9$ & 26 & $29 \cdot 9$ & 137 & $25 \cdot 2$ & 149 & $31 \cdot 1$ \\
\hline Current & 1091 & $27 \cdot 0$ & 133 & $30 \cdot 3$ & 20 & $21 \cdot 7$ & 110 & $27 \cdot 2$ & 110 & $28 \cdot 1$ & 63 & $24 \cdot 1$ & 25 & $28 \cdot 7$ & 126 & $23 \cdot 2$ & 134 & $28 \cdot 0$ \\
\hline Leisure-time PA (\%) & 2771 & $68 \cdot 5$ & 283 & $64 \cdot 5$ & 68 & $73 \cdot 9$ & 189 & $46 \cdot 8$ & 162 & $41 \cdot 4$ & 141 & $53 \cdot 8$ & 40 & $46 \cdot 0$ & 308 & $56 \cdot 7$ & 200 & $41 \cdot 8$ \\
\hline BMI $\left(\mathrm{kg} / \mathrm{m}^{2}\right)$ & $25 \cdot 6$ & $4 \cdot 6$ & $24 \cdot 8$ & $4 \cdot 1$ & $24 \cdot 9$ & $4 \cdot 6$ & $27 \cdot 4$ & $4 \cdot 7$ & $26 \cdot 7$ & 4 & $26 \cdot 9$ & $4 \cdot 3$ & $28 \cdot 5$ & $4 \cdot 4$ & $25 \cdot 3$ & $4 \cdot 6$ & $25 \cdot 8$ & $4 \cdot 3$ \\
\hline BMI class (\%) & & & & & & & & & & & & & & & & & & \\
\hline Normal & 2045 & $50 \cdot 5$ & 248 & $56 \cdot 5$ & 49 & $53 \cdot 3$ & 124 & $30 \cdot 7$ & 149 & $38 \cdot 1$ & 96 & $36 \cdot 6$ & 15 & $17 \cdot 2$ & 302 & $55 \cdot 6$ & 218 & $45 \cdot 5$ \\
\hline Overweight & 1401 & $34 \cdot 6$ & 148 & $33 \cdot 7$ & 32 & $34 \cdot 8$ & 194 & $48 \cdot 0$ & 168 & $43 \cdot 0$ & 112 & $42 \cdot 8$ & 46 & $52 \cdot 9$ & 171 & $31 \cdot 5$ & 191 & $39 \cdot 9$ \\
\hline Obese & 600 & $14 \cdot 8$ & 43 & $9 \cdot 8$ & 11 & $12 \cdot 0$ & 86 & $21 \cdot 3$ & 74 & $18 \cdot 9$ & 54 & $20 \cdot 6$ & 26 & $29 \cdot 9$ & 70 & $12 \cdot 9$ & 70 & $14 \cdot 6$ \\
\hline Waist circumference $(\mathrm{cm})$ & $89 \cdot 4$ & $13 \cdot 7$ & $86 \cdot 4$ & $13 \cdot 0$ & $93 \cdot 0$ & $13 \cdot 2$ & $86 \cdot 5$ & $12 \cdot 5$ & $89 \cdot 8$ & $12 \cdot 4$ & $88 \cdot 9$ & $11 \cdot 4$ & $92 \cdot 9$ & $11 \cdot 5$ & $87 \cdot 0$ & $13 \cdot 1$ & $87 \cdot 3$ & $12 \cdot 2$ \\
\hline Abdominal obesity (\%) & 1145 & $28 \cdot 3$ & 29 & $31 \cdot 5$ & 135 & $33 \cdot 4$ & 91 & $20 \cdot 7$ & 68 & $26 \cdot 0$ & 82 & $21 \cdot 0$ & 27 & $31 \cdot 0$ & 126 & $23 \cdot 2$ & 106 & $22 \cdot 1$ \\
\hline
\end{tabular}

SHS, Swiss Health Survey; PA, physical activity.

Results are expressed as number of participants and percentage, or mean and standard deviation.

Statistical analysis was carried out using the $\chi^{2}$ test or ANOVA; all comparisons are significant at $P<0.001$.

${ }^{*}$ For 2007 only. 
Table 2 Multivariate analysis of the factors associated with overweight and obesity: SHS 1992-2007 and the CoLaus Study

\begin{tabular}{|c|c|c|c|c|c|c|c|c|}
\hline \multirow[b]{3}{*}{ Characteristic } & \multicolumn{4}{|c|}{ Obesity } & \multicolumn{4}{|c|}{ Overweight and obesity } \\
\hline & \multicolumn{2}{|c|}{ SHS ( $n$ 63766) } & \multicolumn{2}{|c|}{ CoLaus (n 6188) } & \multicolumn{2}{|c|}{ SHS ( $n$ 63766) } & \multicolumn{2}{|c|}{ CoLaus (n 6188) } \\
\hline & OR & $95 \% \mathrm{Cl}$ & OR & $95 \% \mathrm{Cl}$ & OR & $95 \% \mathrm{Cl}$ & OR & $95 \% \mathrm{Cl}$ \\
\hline Age (years) & 1.02 & $1 \cdot 01,1 \cdot 03$ & 1.03 & $1 \cdot 02,1 \cdot 04$ & 1.03 & $1 \cdot 02,1 \cdot 04$ & 1.03 & $1 \cdot 02,1 \cdot 04$ \\
\hline \multicolumn{9}{|l|}{ Study year } \\
\hline 1992 & 1.00 & Ref. & - & - & 1.00 & Ref. & - & - \\
\hline 1997 & $1 \cdot 05$ & $0 \cdot 94,1 \cdot 16$ & - & - & $1 \cdot 11$ & $1 \cdot 04,1 \cdot 17$ & - & - \\
\hline 2002 & $1 \cdot 44$ & $1 \cdot 32,1 \cdot 58$ & - & - & $1 \cdot 33$ & $1 \cdot 27,1 \cdot 40$ & - & - \\
\hline 2007 & $1 \cdot 62$ & $1 \cdot 48,1 \cdot 77$ & - & - & $1 \cdot 41$ & $1 \cdot 34,1 \cdot 48$ & - & - \\
\hline \multicolumn{9}{|l|}{ Gender } \\
\hline Woman & 1.00 & Ref. & 1.00 & Ref. & 1.00 & Ref. & 1.00 & Ref. \\
\hline Man & $1 \cdot 28$ & $1 \cdot 20,1 \cdot 36$ & $1 \cdot 35$ & $1 \cdot 17,1 \cdot 56$ & $2 \cdot 50$ & $2 \cdot 41,2 \cdot 60$ & $2 \cdot 56$ & $2 \cdot 30,2 \cdot 84$ \\
\hline \multicolumn{9}{|l|}{ Educational level } \\
\hline Basic & $1 \cdot 00$ & Ref. & $1 \cdot 00$ & Ref. & $1 \cdot 00$ & Ref. & $1 \cdot 00$ & Ref. \\
\hline Secondary & 0.66 & $0 \cdot 62,0 \cdot 71$ & $0 \cdot 64$ & $0 \cdot 54,0.77$ & $0 \cdot 70$ & $0 \cdot 67,0.73$ & $0 \cdot 74$ & $0.64,0.85$ \\
\hline University & 0.41 & $0.37,0.46$ & $0 \cdot 33$ & $0.26,0.43$ & 0.54 & $0.51,0.58$ & 0.47 & $0.39,0.56$ \\
\hline \multicolumn{9}{|l|}{ Smoking } \\
\hline Never & $1 \cdot 00$ & Ref. & $1 \cdot 00$ & Ref. & $1 \cdot 00$ & Ref. & $1 \cdot 00$ & Ref. \\
\hline Former & $1 \cdot 34$ & $1 \cdot 25,1 \cdot 44$ & $1 \cdot 01$ & $0 \cdot 86,1 \cdot 18$ & $1 \cdot 21$ & $1 \cdot 16,1 \cdot 27$ & $1 \cdot 06$ & $0.94,1.20$ \\
\hline Current & 0.94 & $0 \cdot 88,1 \cdot 02$ & $0 \cdot 62$ & $0.52,0.75$ & 0.90 & $0.87,0.94$ & 0.67 & $0.59,0.76$ \\
\hline \multicolumn{9}{|l|}{ Leisure-time PA } \\
\hline No & $1 \cdot 00$ & Ref. & $1 \cdot 00$ & Ref. & $1 \cdot 00$ & Ref. & $1 \cdot 00$ & Ref. \\
\hline Yes & 0.73 & $0 \cdot 68,0.78$ & 0.57 & $0.49,0.65$ & 0.90 & $0.86,0.93$ & 0.63 & $0.56,0.70$ \\
\hline \multicolumn{9}{|l|}{ Nationality } \\
\hline Swiss & $1 \cdot 00$ & Ref. & $1 \cdot 00$ & Ref. & $1 \cdot 00$ & Ref. & $1 \cdot 00$ & Ref. \\
\hline German & $0 \cdot 75$ & $0.57,0.98$ & $0 \cdot 86$ & $0 \cdot 45,1 \cdot 64$ & $0 \cdot 80$ & $0 \cdot 70,0.92$ & $1 \cdot 08$ & $0 \cdot 70,1.67$ \\
\hline Italian & $1 \cdot 28$ & $1 \cdot 12,1 \cdot 47$ & 1.03 & $0 \cdot 79,1 \cdot 35$ & $1 \cdot 45$ & $1 \cdot 33,1 \cdot 58$ & $1 \cdot 63$ & $1 \cdot 29,2 \cdot 06$ \\
\hline French & $0 \cdot 79$ & $0 \cdot 56,1 \cdot 12$ & 0.69 & $0.49,0.96$ & $0 \cdot 74$ & $0 \cdot 61,0 \cdot 89$ & $0 \cdot 87$ & $0.71,1.07$ \\
\hline Spanish & $0 \cdot 83$ & $0 \cdot 60,1 \cdot 15$ & $1 \cdot 17$ & $0 \cdot 84,1 \cdot 62$ & $1 \cdot 36$ & $1 \cdot 15,1 \cdot 61$ & 1.54 & $1 \cdot 17,2 \cdot 04$ \\
\hline Portuguese & 0.68 & $0.49,0.95$ & $1 \cdot 01$ & $0 \cdot 74,1 \cdot 37$ & $1 \cdot 25$ & $1 \cdot 06,1 \cdot 47$ & 1.49 & $1 \cdot 16,1 \cdot 91$ \\
\hline Former Yugoslavian & $1 \cdot 65$ & $1 \cdot 29,2 \cdot 11$ & $2 \cdot 31$ & $1 \cdot 41,3 \cdot 77$ & $1 \cdot 98$ & $1 \cdot 69,2 \cdot 32$ & $5 \cdot 34$ & $3 \cdot 00,9 \cdot 50$ \\
\hline Other European & 1.09 & $0 \cdot 89,1 \cdot 33$ & 0.98 & $0 \cdot 74,1 \cdot 29$ & $1 \cdot 19$ & $1 \cdot 06,1 \cdot 33$ & 0.96 & $0 \cdot 79,1 \cdot 17$ \\
\hline Other world & $0 \cdot 82$ & $0 \cdot 60,1 \cdot 12$ & $1 \cdot 05$ & $0 \cdot 79,1 \cdot 40$ & 0.93 & $0 \cdot 79,1 \cdot 09$ & 1.44 & $1 \cdot 17,1 \cdot 77$ \\
\hline
\end{tabular}

SHS, Swiss Health Survey; PA, physical activity; Ref., reference category.

Statistical analysis was carried out using multivariate logistic regression.

\section{Discussion}

To our knowledge, the present study is the most comprehensive one assessing the prevalence of overweight and obesity according to nationality among migrants living in Switzerland. Our data indicate that overweight and obesity are unevenly distributed among migrants in Switzerland, some groups showing higher overweight and obesity levels independently of education, smoking, physical activity or length of residence. Our findings are partly in agreement with those of a previous study ${ }^{(8)}$, which failed to find any relationship between nationality and obesity status, probably because of the smaller sample size.

The highest prevalence of overweight and obesity was found for migrants from the former Republic of Yugoslavia, a finding already reported for adolescents ${ }^{(15)}$. The reasons for such an increase cannot be attributed solely to differences in age, socio-economic status or physical activity, as this increased prevalence remained after multivariate adjustment. It is possible that some migrants associate a fat body to increased wealth ${ }^{(16)}$, but this statement has been challenged ${ }^{(17,18)}$. Hence, other factors such as diet and eventually a differing genetic background await further investigation.
Some discrepancies were found regarding the factors associated with BMI and waist-derived obesity. No associations were found between most nationalities and abdominal obesity, whereas significant associations were found using BMI. Further, Portuguese migrants had a higher OR for overweight and obesity (BMI-derived) and a lower OR for abdominal obesity, although no significant association was found after adjusting for length of residence. Overall, our findings suggest that the relationship between migrant status or nationality and abdominal obesity might be different from that regarding BMIderived obesity, but the reasons for such a discrepancy await further investigation.

Compared with data from their country of origin ${ }^{(1)}$, differing patterns were found: migrants from France, Germany and Spain presented lower levels of overweight and obesity, whereas migrants from Portugal presented higher levels (Fig. 1). A possible explanation might be the socio-economic status of migrants. Indeed, and as reported in Table 1, the Portuguese had a much lower educational level than the Swiss, whereas the French and Germans had a higher level. Nevertheless, the Spanish also had a lower educational level than the Swiss, but their obesity levels were actually lower than those 
Table 3 Multivariate analysis of the effect of duration on overweight and obesity (migrants only): SHS 2007 and the CoLaus Study

\begin{tabular}{|c|c|c|c|c|c|c|c|c|}
\hline & \multicolumn{4}{|c|}{ Obesity } & \multicolumn{4}{|c|}{ Overweight and obesity } \\
\hline & \multicolumn{2}{|c|}{ SHS 2007 ( $n$ 1832) } & \multicolumn{2}{|c|}{ CoLaus (n 2639) } & \multicolumn{2}{|c|}{ SHS 2007 ( $n$ 1832) } & \multicolumn{2}{|c|}{ CoLaus (n 2639) } \\
\hline & OR & $95 \% \mathrm{Cl}$ & OR & $95 \% \mathrm{Cl}$ & OR & $95 \% \mathrm{Cl}$ & OR & $95 \% \mathrm{Cl}$ \\
\hline Age (years) & $1 \cdot 01$ & $1 \cdot 00,1 \cdot 03$ & $1 \cdot 03$ & $1 \cdot 02,1 \cdot 04$ & $1 \cdot 02$ & $1 \cdot 01,1 \cdot 03$ & $1 \cdot 04$ & $1 \cdot 03,1 \cdot 06$ \\
\hline \multicolumn{9}{|l|}{ Duration of stay (years) } \\
\hline$<10$ & $1 \cdot 00$ & Ref. & $1 \cdot 00$ & Ref. & $1 \cdot 00$ & Ref. & $1 \cdot 00$ & Ref. \\
\hline $10-19$ & $1 \cdot 30$ & $0 \cdot 75,2 \cdot 26$ & 0.91 & $0.59,1.42$ & $1 \cdot 06$ & $0 \cdot 79,1 \cdot 41$ & 0.95 & $0 \cdot 70,1 \cdot 29$ \\
\hline $20-29$ & $2 \cdot 06$ & $1 \cdot 16,3 \cdot 65$ & 0.96 & $0 \cdot 61,1 \cdot 50$ & $1 \cdot 20$ & $0 \cdot 86,1 \cdot 67$ & $0 \cdot 86$ & $0 \cdot 62,1 \cdot 18$ \\
\hline$\geq 30$ & $1 \cdot 84$ & $0.95,3.59$ & $1 \cdot 02$ & $0.63,1.65$ & $1 \cdot 28$ & $0 \cdot 87,1 \cdot 88$ & $0 \cdot 72$ & $0.51,1.01$ \\
\hline \multicolumn{9}{|l|}{ Gender } \\
\hline Woman & $1 \cdot 00$ & Ref. & $1 \cdot 00$ & Ref. & $1 \cdot 00$ & Ref. & $1 \cdot 00$ & Ref. \\
\hline Man & $1 \cdot 18$ & $0 \cdot 84,1 \cdot 65$ & $1 \cdot 11$ & $0.89,1.39$ & $2 \cdot 66$ & $2 \cdot 16,3 \cdot 28$ & $2 \cdot 64$ & $2 \cdot 22,3 \cdot 14$ \\
\hline \multicolumn{9}{|l|}{ Educational level } \\
\hline Basic & $1 \cdot 00$ & Ref. & 1.00 & Ref. & $1 \cdot 00$ & Ref. & $1 \cdot 00$ & Ref. \\
\hline Secondary & 0.63 & $0.43,0.93$ & 0.62 & $0 \cdot 48,0 \cdot 81$ & 0.54 & $0 \cdot 41,0 \cdot 70$ & $0 \cdot 71$ & $0.57,0.88$ \\
\hline University & 0.46 & $0 \cdot 28,0 \cdot 78$ & 0.38 & $0.26,0.55$ & 0.40 & $0.29,0.56$ & 0.50 & $0.38,0.66$ \\
\hline \multicolumn{9}{|l|}{ Smoking } \\
\hline Never & $1 \cdot 00$ & Ref. & $1 \cdot 00$ & Ref. & $1 \cdot 00$ & Ref. & $1 \cdot 00$ & Ref. \\
\hline Former & $1 \cdot 36$ & $0.93,2 \cdot 01$ & 0.97 & $0 \cdot 76,1 \cdot 26$ & $1 \cdot 03$ & $0 \cdot 79,1 \cdot 33$ & $1 \cdot 11$ & $0.91,1.37$ \\
\hline Current & 0.67 & $0.44,1 \cdot 02$ & 0.58 & $0.43,0.77$ & 0.72 & $0.56,0.91$ & $0 \cdot 61$ & $0.50,0.75$ \\
\hline \multicolumn{9}{|l|}{ Leisure-time PA } \\
\hline No & $1 \cdot 00$ & Ref. & $1 \cdot 00$ & Ref. & $1 \cdot 00$ & Ref. & $1 \cdot 00$ & Ref. \\
\hline Yes & 0.64 & $0.46,0.90$ & 0.66 & $0.53,0.83$ & 0.87 & $0 \cdot 70,1 \cdot 07$ & $0 \cdot 71$ & $0.59,0.84$ \\
\hline \multicolumn{9}{|l|}{ Nationality } \\
\hline German & $1 \cdot 00$ & Ref. & $1 \cdot 00$ & Ref. & $1 \cdot 00$ & Ref. & $1 \cdot 00$ & Ref. \\
\hline Italian & $1 \cdot 32$ & $0 \cdot 72,2 \cdot 44$ & $1 \cdot 35$ & $0 \cdot 67,2 \cdot 73$ & $1 \cdot 33$ & $0.92,1.93$ & 1.63 & $0.99,2.67$ \\
\hline French & 0.59 & $0 \cdot 25,1 \cdot 39$ & $0 \cdot 88$ & $0 \cdot 43,1 \cdot 81$ & 0.98 & $0 \cdot 66,1 \cdot 47$ & 0.79 & $0 \cdot 49,1 \cdot 28$ \\
\hline Spanish & $1 \cdot 01$ & $0 \cdot 39,2 \cdot 63$ & 1.57 & $0 \cdot 76,3 \cdot 25$ & $1 \cdot 30$ & $0 \cdot 73,2 \cdot 34$ & 1.51 & $0.90,2 \cdot 53$ \\
\hline Portuguese & $1 \cdot 11$ & $0.53,2 \cdot 36$ & $1 \cdot 50$ & $0 \cdot 71,3 \cdot 15$ & $1 \cdot 16$ & $0 \cdot 74,1 \cdot 81$ & $1 \cdot 35$ & $0 \cdot 80,2 \cdot 27$ \\
\hline Former Republic of Yugoslavia & $2 \cdot 31$ & $1 \cdot 12,4 \cdot 77$ & 3.51 & $1 \cdot 54,8 \cdot 00$ & $2 \cdot 05$ & $1 \cdot 29,3 \cdot 28$ & $5 \cdot 66$ & $2 \cdot 66,12 \cdot 1$ \\
\hline Other European & $1 \cdot 20$ & $0 \cdot 66,2 \cdot 16$ & $1 \cdot 28$ & $0.63,2.57$ & $1 \cdot 17$ & $0.84,1 \cdot 62$ & $0 \cdot 86$ & $0.54,1 \cdot 38$ \\
\hline Other world & $1 \cdot 08$ & $0 \cdot 52,2 \cdot 26$ & $1 \cdot 47$ & $0 \cdot 72,2 \cdot 98$ & $1 \cdot 07$ & $0.72,1 \cdot 59$ & $1 \cdot 30$ & $0 \cdot 81,2 \cdot 11$ \\
\hline
\end{tabular}

SHS, Swiss Health Survey; PA, physical activity; Ref., reference category.

Statistical analysis was carried out using multivariate logistic regression.

Table 4 Trends in the prevalence of overweight and obesity according to survey year (migrants): SHS 1992-2007

\begin{tabular}{|c|c|c|c|c|c|c|c|c|c|c|}
\hline & \multicolumn{2}{|c|}{1992} & \multicolumn{2}{|c|}{1997} & \multicolumn{2}{|c|}{2002} & \multicolumn{2}{|c|}{2007} & \multirow[b]{2}{*}{ Test for trend } & \multirow[b]{2}{*}{$P$ value } \\
\hline & $\%$ & SD & $\%$ & SD & $\%$ & SD & $\%$ & SD & & \\
\hline Germany & $22 \cdot 7$ & $2 \cdot 7$ & $25 \cdot 3$ & $3 \cdot 2$ & 33.9 & $2 \cdot 7$ & $32 \cdot 0$ & $2 \cdot 4$ & $2 \cdot 89$ & $<0.005$ \\
\hline Italy & $39 \cdot 2$ & 1.9 & $47 \cdot 4$ & 1.9 & $48 \cdot 5$ & 1.9 & $52 \cdot 4$ & $2 \cdot 3$ & $4 \cdot 42$ & $<0.001$ \\
\hline France & $17 \cdot 0$ & $2 \cdot 9$ & $27 \cdot 1$ & $4 \cdot 0$ & $29 \cdot 5$ & $3 \cdot 7$ & $33 \cdot 8$ & $3 \cdot 3$ & 3.62 & $<0.001$ \\
\hline Spain & $33 \cdot 7$ & $3 \cdot 5$ & $44 \cdot 1$ & $4 \cdot 0$ & $47 \cdot 1$ & $3 \cdot 8$ & $40 \cdot 8$ & $4 \cdot 8$ & $1 \cdot 78$ & 0.08 \\
\hline Portugal & $32 \cdot 1$ & $4 \cdot 1$ & $29 \cdot 4$ & $3 \cdot 3$ & $48 \cdot 5$ & 3.9 & 43.9 & $3 \cdot 4$ & $3 \cdot 24$ & $<0.002$ \\
\hline Former Republic of Yugoslavia & $42 \cdot 8$ & $4 \cdot 2$ & $44 \cdot 7$ & $3 \cdot 7$ & $48 \cdot 8$ & 3.5 & $50 \cdot 9$ & 3.9 & $1 \cdot \overline{61}$ & $0 \cdot 11$ \\
\hline Other European & $32 \cdot 4$ & $2 \cdot 7$ & $30 \cdot 2$ & $2 \cdot 8$ & $40 \cdot 9$ & $2 \cdot 5$ & $40 \cdot 2$ & $2 \cdot 2$ & $2 \cdot 91$ & $<0.005$ \\
\hline Other world & $19 \cdot 1$ & $3 \cdot 2$ & $28 \cdot \overline{9}$ & $3 \cdot 4$ & $29 \cdot 4$ & $3 \cdot 3$ & $32 \cdot \overline{4}$ & $2 \cdot 8$ & $2 \cdot 66$ & $<0.01$ \\
\hline
\end{tabular}

SHS, Swiss Health Survey.

Test for trend was performed separately for each nationality using the Cochran-Armitage test.

reported for Spain. Although socio-economic status might partly explain the differences in overweight and obesity prevalence between migrants, other factors such as diet should also be considered ${ }^{(19)}$. For instance, and as reported for Greek migrants in Australia ${ }^{(20)}$, Portuguese migrants might adopt a less healthy diet than in their original countries, whereas maintaining a more traditional lifestyle would protect them against obesity ${ }^{(21)}$. Overall, our data indicate that the prevalence of overweight and obesity among migrants in Switzerland depends on their nationality, and does not obligatorily replicate the obesity pattern of the original country. Further, these differences in the prevalence of overweight and obesity cannot only be ascribed to differences in age, socio-economic status or leisure-time physical activity, and other factors such as diet and eventually a differing genetic background should be assessed.

It has been shown that increased length of residence can be associated with the risk of overweight and obesity ${ }^{(5,22,23)}$, although this statement has been challenged ${ }^{(24)}$. The present analysis shows ambiguous results. The small but significant relationships between length of 
(a)

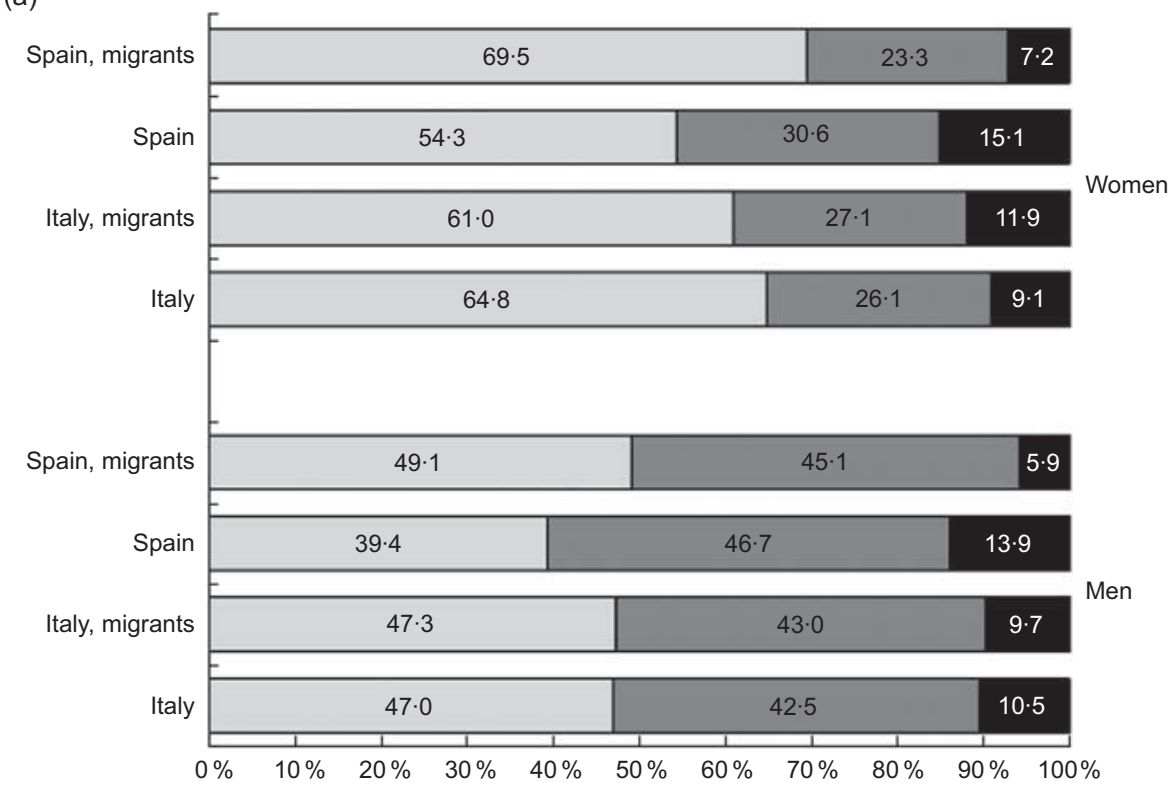

(b)

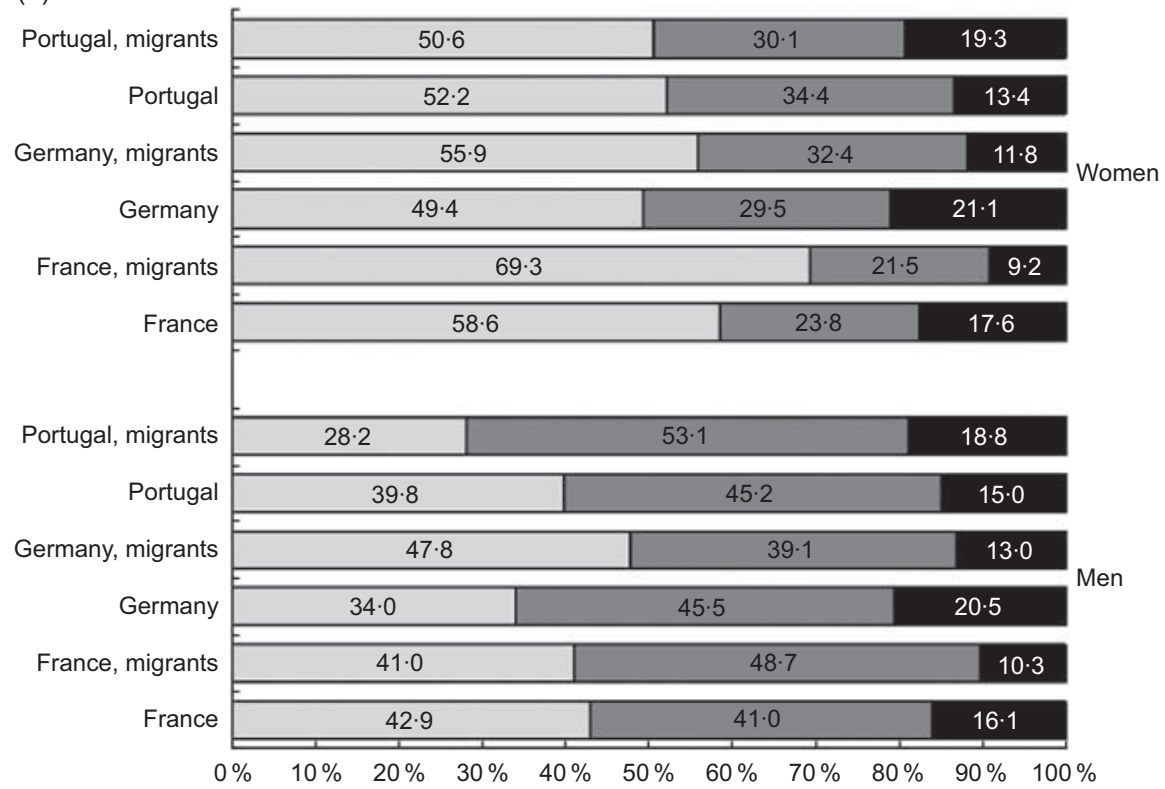

Fig. 1 Comparison of the prevalence of overweight and obesity between migrants and their country of origin: (a) self-reported data and (b) measured data ( $\square$, normal; $\square$, overweight; $\square$, obese)

residence and BMI were no longer significant after multivariate adjustment. Indeed, with increasing length of residence, the obesity rates in migrants tend to increase until they equal or even surpass the host country's rates $^{(25,26)}$. Hence, the risk of becoming obese is likely to depend on the actual prevalence of obesity in the host country; migrants in a country with a high obesity prevalence such as the USA might have a higher risk ${ }^{(5,22)}$ than migrants in a country with a low obesity prevalence such as Sweden ${ }^{(24)}$. Compared with other European countries $^{(1)}$, the prevalence of overweight and obesity in Switzerland is rather low; hence, it is possible that migrants coming to Switzerland may have a lower risk of developing overweight or obesity than migrants going to other countries. Overall, our data indicate that the length of residence does not seem to exert a significant effect on overweight or obesity levels among migrants in Switzerland, possibly because of a less 'obesogenic' environment.

An increase in BMI with time was found for most migrant groups, and this increase tended to be higher than the increase observed for Swiss nationals. However, some increases did not reach statistical significance (i.e. for Spanish and formerly Yugoslavian nationals); the most likely explanation is the relatively small sample sizes, and 
thus limited statistical power. Interestingly, most increases were stronger than those observed for Swiss nationals. A possible explanation is the fact that in some countries the prevalence of overweight and obesity has increased steeper than in Switzerland and that, therefore, migrants who arrived more recently might be more overweight or obese than those who came several years ago.

The present study has several limitations worth pointing out. The participation rate in the CoLaus Study was low (41\%), which might limit the generalization of the findings; however, this participation rate is similar to that of other epidemiological studies ${ }^{(27)}$. In addition, in the SHS, height and weight were self-reported, leading to a probable underestimation of the prevalence of overweight and obesity. Nevertheless, if we assume a non-differential bias, i.e. that the magnitude of height and weight underestimation does not change according to nationality, the relationship between overweight/ obesity and nationality should not change. Finally, specific BMI and waist thresholds were used to define obesity, and it has been suggested that other thresholds should be used for non-Caucasians ${ }^{(28)}$. Nevertheless, as it was not possible to precisely assess the ethnicity of all CoLaus and SHS participants, the use of specific thresholds was justified. The major strength of our study is that we used two population-based samples representative of the Swiss population and that the results obtained were quite similar.

In summary, our results indicate that overweight and obesity are unevenly distributed among migrants in Switzerland. Migrants from Southern Europe and from the former Republic of Yugoslavia present higher prevalence rates. These differences suggest that preventive interventions should be tailored to the needs of specific populations ${ }^{(29)}$.

\section{Acknowledgements}

The CoLaus Study was supported by research grants from GlaxoSmithKline, the Faculty of Biology and Medicine of Lausanne, Switzerland, and the Swiss National Science Foundation (Grant no.: 33CSCO-122661). P.V. and G.W. received an unrestricted grant for GSK to conduct the CoLaus Study. The authors report no conflict of interest. P.M.-V. analysed the data and drafted the manuscript; F.P., G.W. and P.V. conceived and designed the study and revised the manuscript critically for important intellectual content. All authors gave their final approval of the version published. The authors express their gratitude to the participants in the Lausanne CoLaus Study and to the investigators who have contributed to the recruitment, in particular Yolande Barreau, Anne-Lise Bastian, Binasa Ramic, Martine Moranville, Martine Baumer, Marcy Sagette, Jeanne Ecoffey and Sylvie Mermoud for data collection.

\section{References}

1. World Health Organization (2007) The Challenge of Obesity in the WHO European Region and the Strategies for Response. Copenhagen: WHO Regional Office for Europe.

2. Dijkshoorn H, Nierkens V \& Nicolaou M (2008) Risk groups for overweight and obesity among Turkish and Moroccan migrants in The Netherlands. Public Health 122, 625-630.

3. Renzaho AM, Gibbons C, Swinburn B et al. (2006) Obesity and undernutrition in sub-Saharan African immigrant and refugee children in Victoria, Australia. Asia Pac J Clin Nutr 15, 482-490.

4. Wändell PE, Ponzer S, Johansson SE et al. (2004) Country of birth and body mass index: a national study of 2000 immigrants in Sweden. Eur J Epidemiol 19, 1005-1010.

5. Akresh IR (2008) Overweight and obesity among foreignborn and U.S.-born Hispanics. Biodemography Soc Biol $\mathbf{5 4}$, 183-199.

6. Barcenas $\mathrm{CH}$, Wilkinson AV, Strom SS et al. (2007) Birthplace, years of residence in the United States, and obesity among Mexican-American adults. Obesity (Silver Spring) 15, 1043-1052.

7. Office Fédéral des Migrations (2010) Rapport sur la Migration 2009. Berne Wabern, Switzerland: ODM.

8. Bischoff A \& Wanner P (2008) The self-reported health of immigrant groups in Switzerland. J Immigr Minor Health 10, 325-335.

9. Firmann M, Mayor V, Marques-Vidal P et al. (2008) The CoLaus Study: a population-based study to investigate the epidemiology and genetic determinants of cardiovascular risk factors and metabolic syndrome. BMC Cardiovasc Disord 8, 6.

10. Calmonte R, Galati-Petrecca M, Lieberherr R et al. (2005) Gesundheit und Gesundheitsverhalten in der Schweiz 1992-2002. Schweizerische Gesundheitsbefragung. Neuchâtel, Switzerland: BFS

11. Bundesamt für Statistik (2000) Schweizerische Gesundheitsbefragung: Gesundheit und Gesundheitsverhalten in der Schweiz 1997. Neuchâtel, Swtzerland: BFS.

12. IHA-GfK (2003) Schweizerische Gesundheitshefragung SGB 2002 - Schlussbericht zur Datenerbebung. Neuchâtel, Switzerland: BFS

13. Lean ME, Han TS \& Morrison CE (1995) Waist circumference as a measure for indicating need for weight management. BMJ 311, 158-161.

14. Mohadjer L \& Choudhry GH (2001) Adjusting for missing data in low-income surveys. In Studies of Welfare Populations: Data Collection and Research Issues, pp. 129-156 [Panel on Data and Methods for Measuring the Effects of Changes in Social Welfare Programs, M Ver Ploeg, RA Moffitt and CF Citro, editors]. Washington, DC: National Academy Press.

15. Kirchengast S \& Schober E (2006) To be an immigrant: a risk factor for developing overweight and obesity during childhood and adolescence? J Biosoc Sci 38, 695-705.

16. Renzaho AM (2004) Fat, rich and beautiful: changing sociocultural paradigms associated with obesity risk, nutritional status and refugee children from sub-Saharan Africa. Health Place 10, 105-113.

17. Nicolaou M, Doak C, Dam R et al. (2008) Body size preference and body weight perception among two migrant groups of non-Western origin. Public Health Nutr 11, 1332-1341.

18. Råberg M, Kumar B, Holmboe-Ottesen G et al. (2010) Overweight and weight dissatisfaction related to socioeconomic position, integration and dietary indicators among south Asian immigrants in Oslo. Public Health Nutr 13, 695-703. 
19. Kouris-Blazos A, Wahlqvist ML, Trichopoulou A et al. (1996) Health and nutritional status of elderly Greek migrants to Melbourne, Australia. Age Ageing 25, 177-189.

20. Landman J \& Cruickshank JK (2001) A review of ethnicity, health and nutrition-related diseases in relation to migration in the United Kingdom. Public Health Nutr 4, 647-657.

21. Renzaho AM, Swinburn B \& Burns C (2008) Maintenance of traditional cultural orientation is associated with lower rates of obesity and sedentary behaviours among African migrant children to Australia. Int J Obes (Lond) 32, 594-600.

22. Goel MS, McCarthy EP, Phillips RS et al. (2004) Obesity among US immigrant subgroups by duration of residence. JAMA 292, 2860-2867.

23. Gentilucci UV, Picardi A, Manfrini S et al. (2008) Westernization of the Filipino population resident in Rome: obesity, diabetes and hypertension. Diabetes Metab Res Rev 24, 364-370.

24. Bjerregaard P, Jørgensen ME, Andersen S et al. (2002) Decreasing overweight and central fat patterning with
Westernization among the Inuit in Greenland and Inuit migrants. Int J Obes Relat Metab Disord 26, 1503-1510.

25. Greenberg L, Cwikel J \& Mirsky J (2007) Cultural correlates of eating attitudes: a comparison between native-born and immigrant university students in Israel. Int J Eat Disord $\mathbf{4 0}$, $51-58$.

26. Kaplan MS, Huguet N, Newsom JT et al. (2004) The association between length of residence and obesity among Hispanic immigrants. Am J Prev Med 27, 323-326.

27. Grøtvedt L, Kuulasmaa K, Tolonen H et al. (2008) Sampling and recruitment. In Review of Health Examination Surveys in Europe, pp. 82-126 [H Tolonen, P Koponen, A Aromaa et al., editors]. Helsinki, Finland: KTL - National Public Health Institute.

28. Deurenberg P (2001) Universal cut-off BMI points for obesity are not appropriate. Br J Nutr 85, 135-136.

29. Office Fédéral de la Santé Publique (2007) Stratégie Migration et Santé (Phase II: 2008-2013). Bern, Switzerland: OFSP. 